CERN-PPE/95-65

2 May 1995

\title{
Measurements of the b Baryon Lifetime
}

\author{
The ALEPH Collaboration
}

\begin{abstract}
Using about 1.5 million hadronic $Z$ decays recorded with the ALEPH detector, the lifetime of the $\mathrm{b}$ baryons has been measured using two independent data samples. From a maximum likelihood fit to the impact parameter distribution of leptons in $519 \Lambda \ell^{-}$combinations containing a b baryon sample of 290 decays, the measured b baryon lifetime is

$$
\tau_{\text {b-baryon }}=1.05_{-0.11}^{+0.12}(\text { stat }) \pm 0.09(\text { syst }) \text { ps. }
$$

The lifetime of the $\Lambda_{\mathrm{b}}^{0}$ baryon from a maximum likelihood fit to the proper time distribution of $58 \Lambda_{c}^{+} \ell^{-}$candidates containing a $\Lambda_{\mathrm{b}}^{0}$ sample of 44 decays, is

$$
\tau_{\Lambda_{\mathrm{b}}^{0}}=1.02_{-0.18}^{+0.23} \text { (stat) } \pm 0.06(\text { syst }) \text { ps. }
$$
\end{abstract}

(Submitted to Physics Letters B) 


\section{The ALEPH Collaboration}

D. Buskulic, D. Casper, I. De Bonis, D. Decamp, P. Ghez, C. Goy, J.-P. Lees, M.-N. Minard, P. Odier, B. Pietrzyk

Laboratoire de Physique des Particules (LAPP), IN ${ }^{2} P^{3}-C N R S, 74019$ Annecy-le-Vieux Cedex, France

F. Ariztizabal, M. Chmeissani, J.M. Crespo, I. Efthymiopoulos, E. Fernandez, M. Fernandez-Bosman, V. Gaitan, L1. Garrido, ${ }^{15}$ M. Martinez, S. Orteu, A. Pacheco, C. Padilla, F. Palla, A. Pascual, J.A. Perlas, F. Sanchez, F. Teubert

Institut de Fisica d'Altes Energies, Universitat Autonoma de Barcelona, 08193 Bellaterra (Barcelona), Spain ${ }^{7}$

A. Colaleo, D. Creanza, M. de Palma, A. Farilla, G. Gelao, M. Girone, G. Iaselli, G. Maggi, ${ }^{3}$ N. Marinelli, S. Natali, S. Nuzzo, A. Ranieri, G. Raso, F. Romano, F. Ruggieri, G. Selvaggi, L. Silvestris, P. Tempesta, G. Zito

Dipartimento di Fisica, INFN Sezione di Bari, 70126 Bari, Italy

X. Huang, J. Lin, Q. Ouyang, T. Wang, Y. Xie, R. Xu, S. Xue, J. Zhang, L. Zhang, W. Zhao

Institute of High-Energy Physics, Academia Sinica, Beijing, The People's Republic of China ${ }^{8}$

G. Bonvicini, D. Cassel, ${ }^{30}$ M. Cattaneo, P. Comas, P. Coyle, H. Drevermann, A. Engelhardt, R.W. Forty, M. Frank, R. Hagelberg, J. Harvey, R. Jacobsen, ${ }^{24}$ P. Janot, B. Jost, J. Knobloch, I. Lehraus, M. Maggi, C. Markou, ${ }^{23}$ E.B. Martin, P. Mato, H. Meinhard, A. Minten, R. Miquel, T. Oest, P. Palazzi, J.R. Pater, ${ }^{27}$ J.-F. Pusztaszeri, F. Ranjard, P. Rensing, L. Rolandi, D. Schlatter, M. Schmelling, O. Schneider, W. Tejessy, I.R. Tomalin, A. Venturi, H. Wachsmuth, W. Wiedenmann, T. Wildish, W. Witzeling, J. Wotschack

European Laboratory for Particle Physics (CERN), 1211 Geneva 23, Switzerland

Z. Ajaltouni, M. Bardadin-Otwinowska, ${ }^{2}$ A. Barres, C. Boyer, A. Falvard, P. Gay, C. Guicheney, P. Henrard, J. Jousset, B. Michel, S. Monteil, J-C. Montret, D. Pallin, P. Perret, F. Podlyski, J. Proriol, J.-M. Rossignol, F. Saadi

Laboratoire de Physique Corpusculaire, Université Blaise Pascal, IN $P^{3}$-CNRS, ClermontFerrand, 63177 Aubière, France

T. Fearnley, J.B. Hansen, J.D. Hansen, J.R. Hansen, P.H. Hansen, B.S. Nilsson

Niels Bohr Institute, 2100 Copenhagen, Denmark ${ }^{9}$

A. Kyriakis, E. Simopoulou, I. Siotis, A. Vayaki, K. Zachariadou 
Nuclear Research Center Demokritos (NRCD), Athens, Greece

A. Blondel, ${ }^{21}$ G. Bonneaud, J.C. Brient, P. Bourdon, L. Passalacqua, A. Rougé, M. Rumpf, R. Tanaka, A. Valassi, M. Verderi, H. Videau

Laboratoire de Physique Nucléaire et des Hautes Energies, Ecole Polytechnique, IN $\mathrm{I}^{2} \mathrm{P}_{-}$ CNRS, 91128 Palaiseau Cedex, France

D.J. Candlin, M.I. Parsons

Department of Physics, University of Edinburgh, Edinburgh EH9 3JZ, United Kingdom ${ }^{10}$

E. Focardi, G. Parrini

Dipartimento di Fisica, Università di Firenze, INFN Sezione di Firenze, 50125 Firenze, Italy

M. Corden, M. Delfino, ${ }^{12}$ C. Georgiopoulos, D.E. Jaffe

Supercomputer Computations Research Institute, Florida State University, Tallahassee, FL $32306-4052$, USA $^{13,14}$

A. Antonelli, G. Bencivenni, G. Bologna, ${ }^{4}$ F. Bossi, P. Campana, G. Capon, V. Chiarella, G. Felici, P. Laurelli, G. Mannocchi, ${ }^{5}$ F. Murtas, G.P. Murtas, M. Pepe-Altarelli

Laboratori Nazionali dell'INFN (LNF-INFN), 00044 Frascati, Italy

S.J. Dorris, A.W. Halley, I. ten Have, ${ }^{6}$ I.G. Knowles, J.G. Lynch, W.T. Morton, V. O’Shea, C. Raine, P. Reeves, J.M. Scarr, K. Smith, M.G. Smith, A.S. Thompson, F. Thomson, S. Thorn, R.M. Turnbull

Department of Physics and Astronomy, University of Glasgow, Glasgow G12 8QQ,United Kingdom ${ }^{10}$

U. Becker, O. Braun, C. Geweniger, G. Graefe, P. Hanke, V. Hepp, E.E. Kluge, A. Putzer, B. Rensch, M. Schmidt, J. Sommer, H. Stenzel, K. Tittel, S. Werner, M. Wunsch

Institut für Hochenergiephysik, Universität Heidelberg, 69120 Heidelberg, Fed. Rep. of Germany ${ }^{16}$

R. Beuselinck, D.M. Binnie, W. Cameron, D.J. Colling, P.J. Dornan, N. Konstantinidis, L. Moneta, A. Moutoussi, J. Nash, G. San Martin, J.K. Sedgbeer, A.M. Stacey

Department of Physics, Imperial College, London SW7 2BZ, United Kingdom ${ }^{10}$

G. Dissertori, P. Girtler, E. Kneringer, D. Kuhn, G. Rudolph

Institut für Experimentalphysik, Universität Innsbruck, 6020 Innsbruck, Austria ${ }^{18}$ 
C.K. Bowdery, T.J. Brodbeck, P. Colrain, G. Crawford, A.J. Finch, F. Foster, G. Hughes, T. Sloan, E.P. Whelan, M.I. Williams

Department of Physics, University of Lancaster, Lancaster LA1 4YB, United Kingdom ${ }^{10}$

A. Galla, A.M. Greene, K. Kleinknecht, G. Quast, J. Raab, B. Renk, H.-G. Sander, R. Wanke, C. Zeitnitz

Institut für Physik, Universität Mainz, 55099 Mainz, Fed. Rep. of Germany ${ }^{16}$

J.J. Aubert, A.M. Bencheikh, C. Benchouk, A. Bonissent, ${ }^{21}$ G. Bujosa, D. Calvet, J. Carr, C. Diaconu, F. Etienne, M. Thulasidas, D. Nicod, P. Payre, D. Rousseau, M. Talby

Centre de Physique des Particules, Faculté des Sciences de Luminy, IN ${ }^{2} P^{3}$ CNRS, 13288 Marseille, France

I. Abt, R. Assmann, C. Bauer, W. Blum, D. Brown, ${ }^{24}$ H. Dietl, F. Dydak, ${ }^{21}$ G. Ganis, C. Gotzhein, K. Jakobs, H. Kroha, G. Luitjens, G. Lutz, W. Männer, H.-G. Moser, R. Richter, A. Rosado-Schlosser, R. Settles, H. Seywerd, U. Stierlin, ${ }^{2}$ R. St. Denis, G. Wolf

Max-Planck-Institut für Physik, Werner-Heisenberg-Institut, 80805 München, Fed. Rep. of Germany ${ }^{16}$

R. Alemany, J. Boucrot, O. Callot, A. Cordier, F. Courault, M. Davier, L. Duflot, J.F. Grivaz, Ph. Heusse, M. Jacquet, D.W. Kim ${ }^{19}$ F. Le Diberder, J. Lefrançois, A.-M. Lutz, G. Musolino, I. Nikolic, H.J. Park, I.C. Park, M.-H. Schune, S. Simion, J.-J. Veillet, I. Videau

Laboratoire de l'Accélérateur Linéaire, Université de Paris-Sud, IN ${ }^{2} P^{3}-C N R S, 91405$ Orsay Cedex, France

D. Abbaneo, P. Azzurri, G. Bagliesi, G. Batignani, S. Bettarini, C. Bozzi, G. Calderini, M. Carpinelli, M.A. Ciocci, V. Ciulli, R. Dell'Orso, R. Fantechi, I. Ferrante, L. Foà, ${ }^{1}$ F. Forti, D. Gambino, A. Giassi, M.A. Giorgi, A. Gregorio, F. Ligabue, A. Lusiani, P.S. Marrocchesi, A. Messineo, G. Rizzo, G. Sanguinetti, A. Sciabà, P. Spagnolo, J. Steinberger, R. Tenchini, G. Tonelli, ${ }^{26}$ G. Triggiani, C. Vannini, P.G. Verdini, J. Walsh

Dipartimento di Fisica dell'Università, INFN Sezione di Pisa, e Scuola Normale Superiore, 56010 Pisa, Italy

A.P. Betteridge, G.A. Blair, L.M. Bryant, F. Cerutti, Y. Gao, M.G. Green, D.L. Johnson, T. Medcalf, Ll.M. Mir, P. Perrodo, J.A. Strong

Department of Physics, Royal Holloway \& Bedford New College, University of London, Surrey TW20 OEX, United Kingdom ${ }^{10}$

V. Bertin, D.R. Botterill, R.W. Clifft, T.R. Edgecock, S. Haywood, M. Edwards, P. Maley, P.R. Norton, J.C. Thompson 
Particle Physics Dept., Rutherford Appleton Laboratory, Chilton, Didcot, Oxon OX11 OQX, United Kingdom ${ }^{10}$

B. Bloch-Devaux, P. Colas, H. Duarte, S. Emery, W. Kozanecki, E. Lançon, M.C. Lemaire, E. Locci, B. Marx, P. Perez, J. Rander, J.-F. Renardy, A. Rosowsky, A. Roussarie, J.P. Schuller, J. Schwindling, D. Si Mohand, A. Trabelsi, B. Vallage

CEA, DAPNIA/Service de Physique des Particules, CE-Saclay, 91191 Gif-sur-Yvette Cedex, France $^{17}$

R.P. Johnson, H.Y. Kim, A.M. Litke, M.A. McNeil, G. Taylor

Institute for Particle Physics, University of California at Santa Cruz, Santa Cruz, CA 95064, $U S A^{22}$

A. Beddall, C.N. Booth, R. Boswell, S. Cartwright, F. Combley, I. Dawson, A. Koksal, M. Letho, W.M. Newton, C. Rankin, L.F. Thompson

Department of Physics, University of Sheffield, Sheffield S3 7RH, United Kingdom ${ }^{10}$

A. Böhrer, S. Brandt, G. Cowan, E. Feigl, C. Grupen, G. Lutters, J. Minguet-Rodriguez, F. Rivera, ${ }^{25}$ P. Saraiva, L. Smolik, F. Stephan

Fachbereich Physik, Universität Siegen, 57068 Siegen, Fed. Rep. of Germany ${ }^{16}$

M. Apollonio, L. Bosisio, R. Della Marina, G. Giannini, B. Gobbo, F. Ragusa ${ }^{20}$

Dipartimento di Fisica, Università di Trieste e INFN Sezione di Trieste, 34127 Trieste, Italy

J. Rothberg, S. Wasserbaech

Experimental Elementary Particle Physics, University of Washington, WA 98195 Seattle, U.S.A.

S.R. Armstrong, L. Bellantoni, P. Elmer, Z. Feng, D.P.S. Ferguson, Y.S. Gao, S. González, J. Grahl, J.L. Harton, ${ }^{28}$ O.J. Hayes, H. Hu, P.A. McNamara III, J.M. Nachtman, W. Orejudos, Y.B. Pan, Y. Saadi, M. Schmitt, I.J. Scott, V. Sharma, ${ }^{29}$ J.D. Turk, A.M. Walsh, Sau Lan Wu, X. Wu, J.M. Yamartino, M. Zheng, G. Zobernig

Department of Physics, University of Wisconsin, Madison, WI 53706, USA ${ }^{11}$ 


\footnotetext{
${ }^{1}$ Now at CERN, 1211 Geneva 23, Switzerland.

${ }^{2}$ Deceased.

${ }^{3}$ Now at Dipartimento di Fisica, Università di Lecce, 73100 Lecce, Italy.

${ }^{4}$ Also Istituto di Fisica Generale, Università di Torino, Torino, Italy.

${ }^{5}$ Also Istituto di Cosmo-Geofisica del C.N.R., Torino, Italy.

${ }^{6}$ Now at TSM Business School, Enschede, The Netherlands.

${ }^{7}$ Supported by CICYT, Spain.

${ }^{8}$ Supported by the National Science Foundation of China.

${ }^{9}$ Supported by the Danish Natural Science Research Council.

${ }^{10}$ Supported by the UK Particle Physics and Astronomy Research Council.

${ }^{11}$ Supported by the US Department of Energy, contract DE-AC02-76ER00881.

${ }^{12}$ On leave from Universitat Autonoma de Barcelona, Barcelona, Spain.

${ }^{13}$ Supported by the US Department of Energy, contract DE-FG05-92ER40742.

${ }^{14}$ Supported by the US Department of Energy, contract DE-FC05-85ER250000.

${ }^{15}$ Permanent address: Universitat de Barcelona, 08208 Barcelona, Spain.

${ }^{16}$ Supported by the Bundesministerium für Forschung und Technologie, Fed. Rep. of Germany.

${ }^{17}$ Supported by the Direction des Sciences de la Matière, C.E.A.

${ }^{18}$ Supported by Fonds zur Förderung der wissenschaftlichen Forschung, Austria.

${ }^{19}$ Permanent address: Kangnung National University, Kangnung, Korea.

${ }^{20}$ Now at Dipartimento di Fisica, Università di Milano, Milano, Italy.

${ }^{21}$ Also at CERN, 1211 Geneva 23, Switzerland.

${ }^{22}$ Supported by the US Department of Energy, grant DE-FG03-92ER40689.

${ }^{23}$ Now at University of Athens, 157-71 Athens, Greece.

${ }^{24}$ Now at Lawrence Berkeley Laboratory, Berkeley, CA 94720, USA.

${ }^{25}$ Partially supported by Colciencias, Colombia.

${ }^{26}$ Also at Istituto di Matematica e Fisica, Università di Sassari, Sassari, Italy.

${ }^{27}$ Now at Schuster Laboratory, University of Manchester, Manchester M13 9PL, UK.

${ }^{28}$ Now at Colorado State University, Fort Collins, CO 80523, USA.

${ }^{29}$ Now at University of California at San Diego, La Jolla, CA 92093, USA.

${ }^{30}$ Permanent address: Newman Laboratory, Cornell University, Ithaca, NY 14853, USA.
} 


\section{Introduction}

Measurements of individual $\mathrm{b}$ hadron lifetimes are of interest because they test present understanding of $b$ hadron decay dynamics. In the simple spectator picture, the lifetimes of the various $b$ hadron species are equal. Deviations from this picture could arise from non-perturbative QCD corrections or the presence of non-spectator effects such as Wexchange or $\mathrm{W}$-annihilation. In contrast to the diversity observed in the lifetimes of charm hadrons, the differences among various $b$ hadron species have been estimated to be not much larger than $10 \%$ [1], due to the larger b quark mass. Non-spectator effects should be small in the case of $b$ mesons, but could be larger in b baryon decays due to the absence of helicity suppression in the final state; thus a comparison of the lifetimes of $b$ mesons and $b$ baryons is of special interest. Measurements at LEP [2] and the Tevatron [3] have confirmed the expected equality of the b meson lifetimes within $10 \%$, but the published measurements of $b$ baryon lifetime [4] have not yet reached the precision necessary for a definitive comparison of $b$ meson and $b$ baryon lifetimes.

Evidence for $\mathrm{b}$ baryons in $\mathrm{Z}$ decays via correlation between a $\Lambda$ and a prompt high transverse momentum lepton has been reported previously by the ALEPH, OPAL and DELPHI collaborations [5]. The signal results from semileptonic b baryon decays ${ }^{1}$, dominated mainly by $\Lambda_{\mathrm{b}}^{0}$ decays $[6,7]$ such as $\Lambda_{\mathrm{b}}^{0} \rightarrow \Lambda_{\mathrm{c}}^{+} \ell^{-} \bar{\nu}$ followed by the decay $\Lambda_{\mathrm{c}}^{+} \rightarrow \Lambda \mathrm{X}$, with the $\Lambda$ decaying to $\mathrm{p} \pi^{-}$. In addition, $\Lambda_{\mathrm{b}}^{0}$ semileptonic decays with the exclusive reconstruction of the $\Lambda_{c}^{+}$i.e. $\Lambda_{\mathrm{b}}^{0} \rightarrow \Lambda_{c}^{+} \ell^{-} \bar{\nu}$ where $\Lambda_{c}^{+} \rightarrow \mathrm{pK}^{-} \pi^{+}$, have also been reported by ALEPH [8]. Although statistically less powerful for $\Lambda_{\mathrm{b}}^{0}$ identification compared with the $\Lambda \ell^{-}$method, this decay channel gives an independent $\Lambda_{\mathrm{b}}^{0}$ sample and has the advantage of being insensitive to other $\mathrm{b}$ baryons ${ }^{2}$.

In this letter, two independent measurements of the b baryon lifetime using $\Lambda \ell^{-}$and $\Lambda_{c}^{+} \ell^{-}$samples are reported, based on about 1.5 million hadronic $\mathrm{Z}$ decays recorded with the ALEPH detector from 1991 to 1993.

\section{The ALEPH detector}

The ALEPH detector has been described in detail elsewhere [9] and only a brief description is given here. Charged tracks in the central polar region are measured by a silicon vertex detector (VDET) [10] which consists of two concentric barrels of microstrip silicon detectors with double-sided readout positioned at average radii of 6.5 and $11.3 \mathrm{~cm}$. The vDET point resolution, as measured with data [11], is $12 \mu \mathrm{m}$ at normal incidence for both $r \phi$ and $r z$ projections. Tracks are also measured over the range $|\cos \theta|<0.95$, where $\theta$ is the polar angle, by an inner cylindrical drift chamber (ITC) and a large cylindrical time projection chamber (TPC). The ITC provides up to 8 coordinates per track in the $r \phi$ view at radii from 16 to $26 \mathrm{~cm}$. The TPC reconstructs up to 21 space coordinates per track at radii between 30 and $180 \mathrm{~cm}$ and also provides up to 338 measurements of the specific ionization $(\mathrm{d} E / \mathrm{d} x$ ) of each charged track. For electrons in hadronic events, the $\mathrm{d} E / \mathrm{d} x$ resolution is $4.5 \%$ for 338 ionization samples. The tracking detectors are immersed in an axial magnetic field of $1.5 \mathrm{~T}$, providing a transverse momentum resolution of $\sigma_{p} / p=0.0006 p(p$ in $\mathrm{GeV} / c)$ for $45 \mathrm{GeV} / c$ muons.

The interaction point is reconstructed on an event-by-event basis with a method [11]

\footnotetext{
${ }^{1}$ Charge conjugate decays are implied throughout this paper.

${ }^{2}$ Semileptonic decays of other b baryons (for example, $\Xi_{\mathrm{b}}$ ) involve, besides the lepton, mainly strange-charmed baryons in the final state.
} 
which takes advantage of precise three dimensional coordinates for charged tracks provided by the VDET and the beam envelope measurement. With this algorithm, the interaction point is known with a resolution of $50 \mu \mathrm{m}$ in the horizontal direction and $10 \mu \mathrm{m}$ in the vertical direction in the plane perpendicular to the beam direction. Using this event-byevent reconstructed interaction point, the $r \phi$ impact parameter resolution for lepton tracks with VDET hits and momenta above $3 \mathrm{GeV} / c$ is about $50 \mu \mathrm{m}$.

The electromagnetic calorimeter (ECAL), which surrounds the TPC and is inside the coil of the superconducting solenoid, is used to measure electromagnetic energy and, together with the $\mathrm{d} E / \mathrm{d} x$ information in TPC, to identify electrons. The hadron calorimeter (HCAL) is composed of the iron of the magnet return yoke interleaved with 23 layers of streamer tubes and is surrounded by the muon chambers, two additional layers of streamer tubes that cover the same angular range as the HCAL. Detailed discussions of lepton identification are given in reference [12].

\section{Measurement of the b baryon lifetime using $\Lambda \ell^{-}$ correlation}

The method of $\Lambda \ell$ correlation to isolate semileptonic decays of $\mathrm{b}$ baryons has been described in previous letters [5]. This method is sensitive to decays of various species of $\mathrm{b}$ baryons which have a $\Lambda \ell^{-}$in the final state. For brevity of notation, throughout this section, the symbols $\Lambda_{\mathrm{b}}$ and $\Lambda_{\mathrm{c}}^{+}$are used to denote not just $\Lambda_{\mathrm{b}}^{0}$ and $\Lambda_{\mathrm{c}}^{+}$but also analogous beauty and charm baryon states such as $\Xi_{\mathrm{b}}$ and $\Xi_{\mathrm{c}}$.

\subsection{Selection of b baryons using $\Lambda \ell^{-}$correlation}

The $\Lambda$ candidates are identified by their decay $\Lambda \rightarrow \mathrm{p} \pi^{-}$, using an algorithm which uses two oppositely charged tracks that have at least four hits in the TPC to form a vertex. To reduce combinatorial background, $\Lambda$ candidates are required to have at least $3 \mathrm{GeV} / c$ of momentum and a decay length of at least $5 \mathrm{~cm}$ with respect to the interaction point. The measurements of track $\mathrm{d} E / \mathrm{d} x$, when available, are also required to be compatible, within three standard deviations, with that for a proton and a pion. To reduce the contamination from other displaced vertices, the invariant mass of the two daughter tracks is required to be incompatible with both the $K_{S}^{0}$ and $\gamma$ mass hypotheses. The lepton candidates are required to have at least one associated $r \phi$ coordinate in the VDET, five hits in the TPC and two hits in the ITC and a $\chi^{2} /$ d.o.f. for the track fit of less than 4 . Lepton and $\Lambda$ candidates are required to be within 45 degrees of each other.

There are seven possible sources of $\Lambda \ell$ combinations

$$
\begin{array}{r}
\Lambda_{\mathrm{b}} \rightarrow \Lambda_{c}^{+} \mathrm{X} \ell^{-} \bar{\nu}, \quad \Lambda_{\mathrm{c}}^{+} \rightarrow \Lambda \mathrm{X} \\
\overline{\mathrm{B}} \rightarrow \Lambda_{c}^{+} \mathrm{X} \ell^{-} \bar{\nu}, \quad \Lambda_{\mathrm{c}}^{+} \rightarrow \Lambda \mathrm{X} \\
\mathrm{b} \rightarrow \Lambda_{\mathrm{c}}^{+} \mathrm{D}_{\mathrm{s}}^{-} \mathrm{X}, \quad \Lambda_{c}^{+} \rightarrow \Lambda \mathrm{X}, \mathrm{D}_{\mathrm{s}}^{-} \rightarrow \mathrm{X} \ell^{-} \bar{\nu} \\
\mathrm{b} \rightarrow \Lambda_{\mathrm{c}}^{+\mathrm{X}}, \quad \Lambda_{c}^{+} \rightarrow \Lambda \ell^{+} \mathrm{X} \\
\mathrm{c} \rightarrow \Lambda_{c}^{+\mathrm{X}}, \quad \Lambda_{c}^{+} \rightarrow \Lambda \ell^{+} \mathrm{X} \\
\text { Accidental combinations } \\
\text { Fake combinations }
\end{array}
$$


The $\mathrm{D}_{\mathrm{s}}^{-}$in process $(3)$ is the dominant charm hadron contribution of the $\mathrm{W}^{-}$decay. The accidental combinations are real $\Lambda$ from fragmentation in association with real or fake leptons. Fake combinations are spurious $\mathrm{p} \pi^{-}$combinations under the $\Lambda$ peak paired with real or fake leptons. By requiring a lepton candidate to have at least $3 \mathrm{GeV} / \mathrm{c}$ of momentum and a transverse momentum $p_{\perp}$ with respect to the associated jet ${ }^{3}$ of at least $1 \mathrm{GeV} / c$, most of the $\Lambda \ell$ combinations from processes (2) to (5) are removed. The remaining $\Lambda \ell^{-}$combinations originate mostly from either b baryon semileptonic decays (1), accidental combinations (6) or fake combinations (7), while the $\Lambda \ell^{+}$combinations are mostly accidental (6) and fake combinations (7).

The $\Lambda \ell^{-}$(right-sign) and $\Lambda \ell^{+}$(wrong-sign) combinations after application of the $\Lambda$ and lepton selection criteria described above are shown in Fig. 1, where the $\mathrm{p} \pi^{-}$invariant mass spectrum is plotted. The two $\mathrm{p} \pi^{-}$invariant mass distributions are fitted using a Gaussian to represent the $\Lambda$ signal and a second order polynomial to represent the shape of the combinatorial background. The resulting mass and r.m.s. of the Gaussian are $1.1156 \pm 0.0001$ and $0.0025 \pm 0.0001 \mathrm{GeV} / c^{2}$ respectively, consistent with the expectations. The areas of the fitted Gaussians yield $441 \pm 29 \Lambda \ell^{-}$combinations and $217 \pm 24 \Lambda \ell^{+}$ combinations.

To estimate the $\mathrm{b}$ baryon fraction in the $\Lambda \ell^{-}$sample, the residual contributions of the background processes are evaluated. Based on a simulation of process $(2)$, the $90 \%$ confidence level upper limit of $\operatorname{Br}\left(\overline{\mathrm{B}} \rightarrow \mathrm{p} e^{-\mathrm{X}}\right) \leq 0.16 \%$ set by the ARGus collaboration [13] and the measured rate $\operatorname{Br}\left(\Lambda_{c}^{+} \rightarrow \mathrm{pX}\right)=(50 \pm 16) \%$ [14], less than $13 \Lambda \ell^{-}$combinations from this process are expected. This possible contribution is included in the systematic error on the measurement of the $b$ baryon production rate and lifetime. A study of about 1.6 million Monte Carlo $\mathrm{Z} \rightarrow \mathrm{q} \overline{\mathrm{q}}$ events simulated with JETSET 7.3 [15] predicts a negligible contribution from process (3). The contribution of processes (4) and (5) to the $\Lambda \ell^{+}$sample is estimated on basis of measured branching ratios [14] to be $28 \pm 8$ combinations. These are subtracted from the wrong-sign peak as a correction to the number of accidental combinations it contains. The ratio of $\Lambda \ell^{-}$to $\Lambda \ell^{+}$accidental combinations is estimated from the JETSET 7.3 simulation program as $0.8 \pm 0.2$. The error reflects an uncertainty in the knowledge of the momentum spectra of the $\Lambda$ baryons produced in the process of $b$ or $\bar{b}$ quark fragmentation.

The excess of $\Lambda \ell^{-}$combinations over $\Lambda \ell^{+}$, after correcting for the imbalance of the right and wrong-sign is ascribed to semileptonic decay of b baryons. The resulting $b$ baryon signal in the $\Lambda \ell^{-}$sample is $290 \pm 35$ (stat) \pm 39 (syst) events.

To calculate the product branching ratio, a detection efficiency of $(7.5 \pm 0.6) \%$ is determined using a Monte Carlo simulation [16] of process (1) in which a b baryon polarization of $-30 \%[17]$ is assumed. Applying this efficiency, the product branching ratio is

$$
B r\left(\mathrm{~b} \rightarrow \Lambda_{\mathrm{b}}\right) \cdot \operatorname{Br}\left(\Lambda_{\mathrm{b}} \rightarrow \Lambda_{c}^{+} \ell^{-} \bar{\nu} \mathrm{X}\right) \cdot \operatorname{Br}\left(\Lambda_{c}^{+} \rightarrow \Lambda \mathrm{X}\right)=(0.61 \pm 0.06(\text { stat }) \pm 0.10 \text { (syst) }) \%
$$

This product branching ratio is the sum of the electron and muon channels, which is consistent with and supersedes ALEPH's published values [4, 5].

\section{$3.2 \mathrm{~b}$ baryon lifetime fit using $\Lambda \ell^{-}$correlation}

The lifetime of the $\mathrm{b}$ baryon is extracted by a maximum likelihood fit to the $\mathrm{r} \phi$ impact parameter distribution of lepton candidates in the $\Lambda \ell^{-}$sample. The fitting procedure is similar to that used in the previous measurement of the b baryon lifetime [4]. In contrast

\footnotetext{
${ }^{3}$ The lepton is included in the calculation of the jet direction.
} 


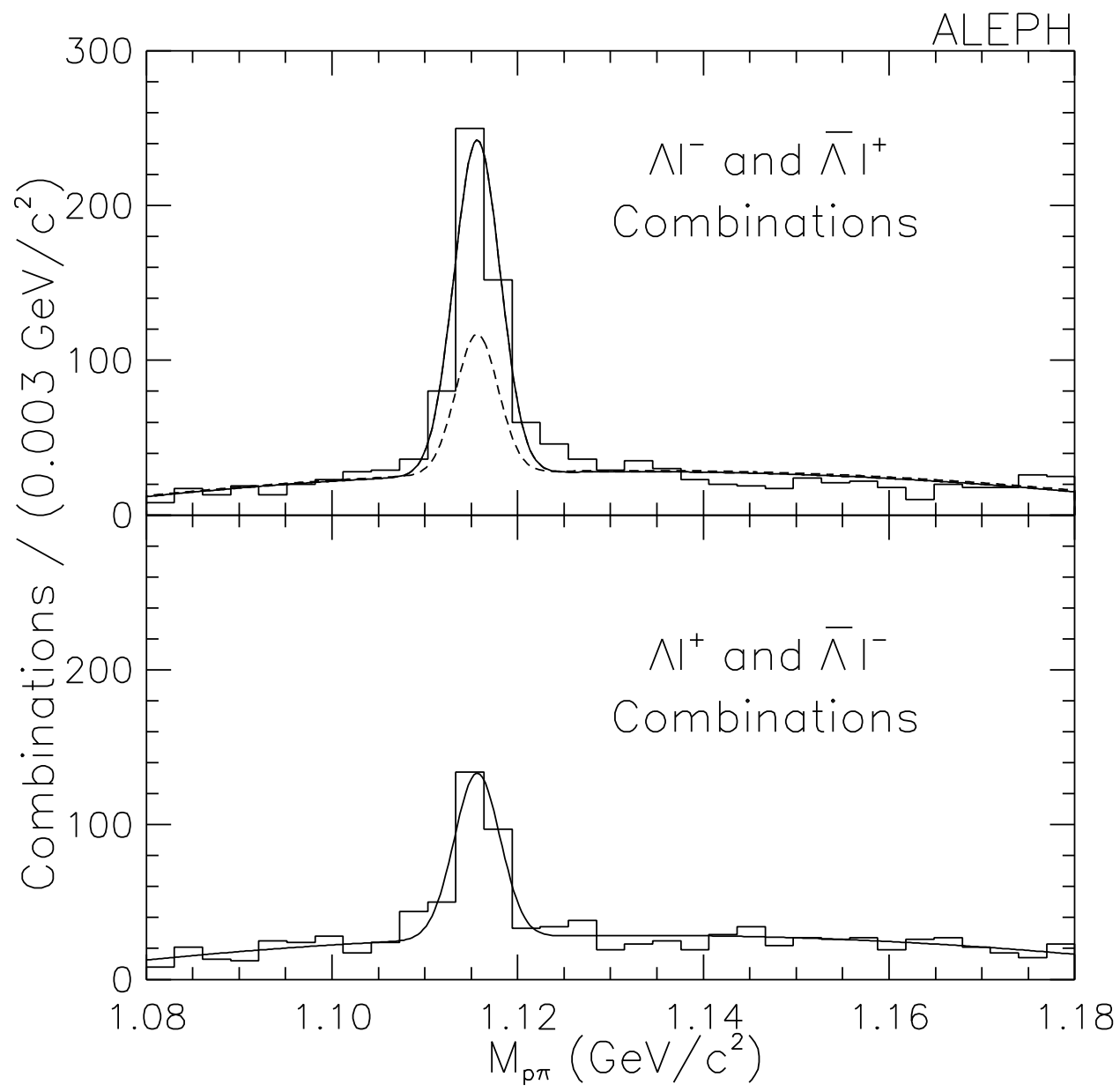

Figure 1: The $\mathrm{p} \pi^{-}$invariant mass distribution of the $\Lambda \ell^{-}$and $\Lambda \ell^{+}$combinations. The dashed curve represents the accidental $\Lambda \ell$ combinations in the $\Lambda \ell^{-}$(right-sign) combinations estimated from the $\Lambda \ell^{+}$(wrong-sign) combinations. 
to the previous measurement, the charged particle trajectory information from the silicon vertex detector is used, both in the reconstruction of the lepton track and in the evaluation, event by event, of the interaction point.

The $\Lambda$ and lepton identification requirements described in the previous subsection, together with a cut of $6 \mathrm{MeV} / c^{2}$ around the $\Lambda$ mass to select the $\mathrm{p} \pi^{-}$combinations, yield a final sample (including the combinatorial (fake) background under the $\Lambda$ mass peak) of $519 \Lambda \ell^{-}$candidates. The b baryon fraction in this sample is about $58 \%$.

In the fit, the lepton impact parameter distribution in $\Lambda \ell^{-}$combinations is described as a sum of different sources of the lepton. Table 1 shows their relative contribution to the $\Lambda \ell^{-}$sample for the average of the electron and muon channels. The fraction of the signal, semileptonic decay of $\mathrm{b}$ baryon $\left(\Lambda_{\mathrm{b}} \rightarrow \ell\right)$, is derived from the ratio of $\Lambda \ell^{-}$and $\Lambda \ell^{+}$combinations observed in the data, corrected for the production asymmetry of the accidental $\Lambda \ell$ pairs. The relative background fractions are taken from simulated events and any possible disagreement with the data is considered in evaluating the systematic error.

The impact parameter distribution for prompt lepton sources (first four components) is obtained by convolving a resolution function with a so-called "physics function" which describes the expected impact parameter for these processes. The physics function is obtained separately for each prompt lepton source from a Monte Carlo simulation of the decay process.

\begin{tabular}{|c|c|}
\hline Lepton source & $\%$ \\
\hline \hline & \\
$\Lambda_{\mathrm{b}} \rightarrow \ell$ & $58 \%$ \\
$\mathrm{~b} \rightarrow \ell$ & $35 \%$ \\
$\mathrm{~b} \rightarrow(\mathrm{c} / \tau) \rightarrow \ell$ & $3.3 \%$ \\
$\mathrm{c} \rightarrow \ell$ & $1.7 \%$ \\
Misidentified hadrons & $1.2 \%$ \\
$\pi$ and K decays & $0.8 \%$ \\
\hline
\end{tabular}

Table 1: Lepton sources in the $\Lambda \ell^{-}$sample.

The physics functions for the $\Lambda_{\mathrm{b}} \rightarrow \ell$ and for the $\mathrm{b} \rightarrow \ell$ component are obtained from Monte Carlo. Two different physics functions are used for the underlying $b \rightarrow \ell$ background, according to the source of the associated $\Lambda$ candidate:

- $\mathrm{b} \rightarrow \ell$ with an accidental $\Lambda$, i.e. a $\Lambda$ coming from fragmentation;

- $\mathrm{b} \rightarrow \ell$ with a fake $\mathrm{p} \pi^{-}$combination.

The difference in these physics functions is due to the different momentum spectra of the " $\Lambda$ " candidates in the two cases.

The signal physics function depends on the unknown b baryon lifetime, the only parameter of the fit, while the physics functions of the $\mathrm{b} \rightarrow \ell$ and $\mathrm{b} \rightarrow \mathrm{c} \rightarrow \ell$ background depend on the average $b$ hadron lifetime. The value used in the fit is a world average of all the $\mathrm{b}$ lifetime measurements $\tau_{\mathrm{b}}=1.537 \pm 0.021 \mathrm{ps}$ [14]. 
The impact parameter resolution function for the leptons is obtained using simulated events. Corrections to resolution function at the level of $10 \%$ are applied to account for the difference in the impact parameter resolution between data and simulated events. This correction is obtained by comparing the negatively-signed part of the impact parameter distribution of hadron tracks in light quark enriched data and Monte Carlo samples, selected by the algorithm described in ref. [18]. The negative side of the impact parameter distribution results mainly from resolution effects.

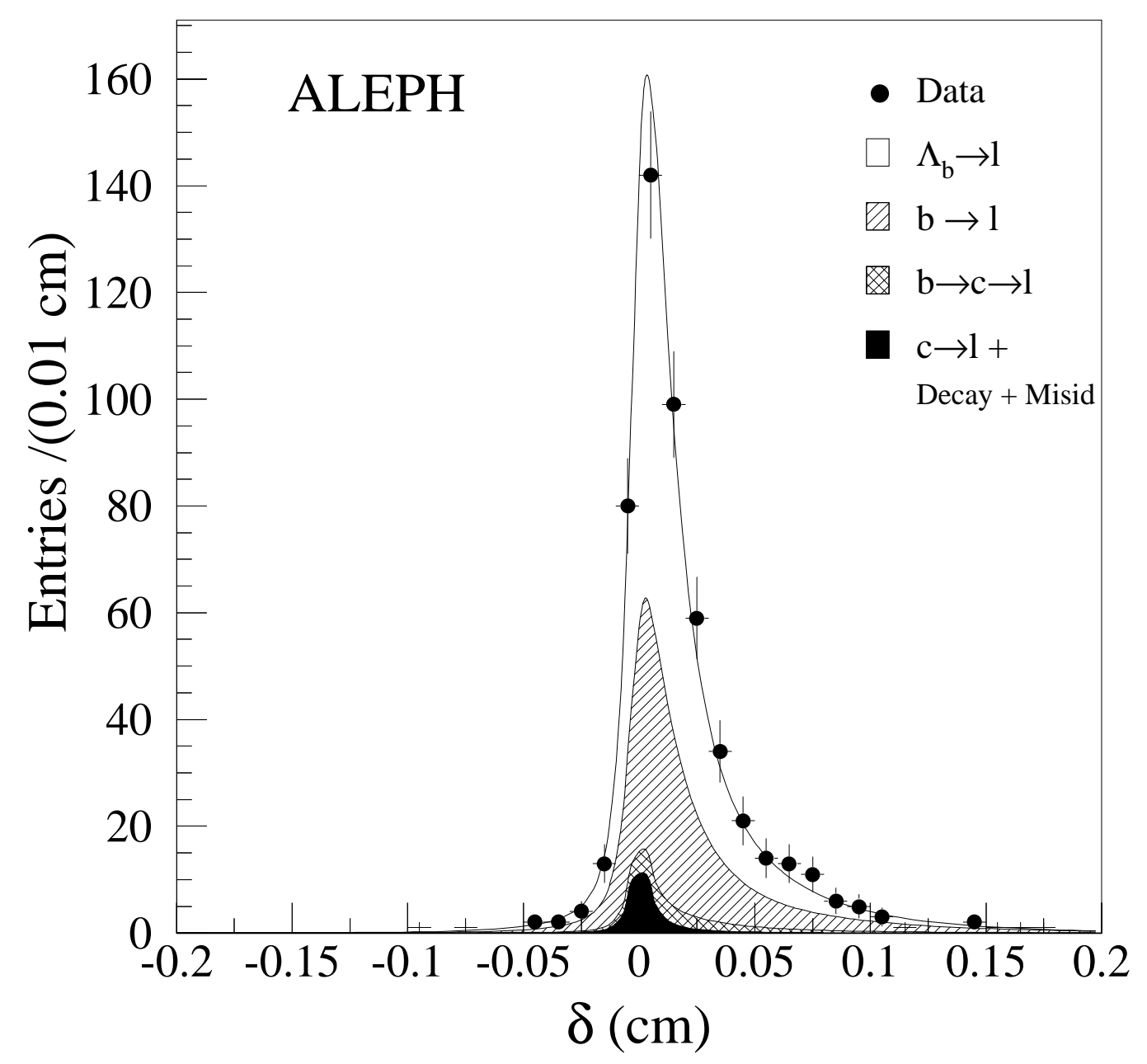

Figure 2: Impact parameter distribution of the selected $\Lambda \ell^{-}$candidates. The solid line is the probability function at the fitted value of the lifetime.

The expected impact parameter distribution for hadrons misidentified as leptons is obtained from the impact parameter distribution of hadrons selected in the data with the same kinematic requirements as applied to the leptons. The distribution for the leptons coming from decay in flight of $\mathrm{K}$ and $\pi$ is taken from the simulated events.

The unbinned maximum likelihood fit to the lepton impact parameter distribution in 
the $519 \Lambda \ell^{-}$candidates yields the b baryon lifetime

$$
\tau_{\mathrm{b}-\text { baryon }}=1.05_{-0.11}^{+0.12} \mathrm{ps},
$$

where the quoted error is statistical. Fig. 2 shows the result of fit together with the observed impact parameter distribution of the lepton candidates in the $\Lambda \ell^{-}$sample. This result supersedes ALEPH's published value [4].

\subsection{Systematic checks}

Several checks for possible biases in the fitted b baryon lifetime value have been performed. First, to check the fitting procedure, the analysis has been applied to a Monte Carlo sample of about 3 million $\mathrm{Z} \rightarrow \mathrm{q} \bar{q}$ events generated with an input $\Lambda_{\mathrm{b}}$ lifetime of $1.5 \mathrm{ps}$. The lifetime fit result is consistent with the input value.

Repeating the fit having varied the kinematic cuts on lepton $p, p_{\perp}$ and the $\Lambda$ momentum, the resulting deviations in the fitted lifetime values are within the range for the given statistical uncertainty.

The background physics functions and the lepton background fractions are checked by fitting the events in the side bands of the $\mathrm{p} \pi^{-}$invariant mass for the average $\mathrm{b}$ hadron lifetime. The fit yields $\tau$ (side band) $=1.52 \pm 0.07 \mathrm{ps}$, in agreement with the average $\mathrm{b}$ hadron lifetime value.

\subsection{Systematic errors}

\begin{tabular}{|l|c|}
\hline & \\
Source of systematic error & Uncertainty (ps) \\
\hline \hline & \\
b baryon fraction $\left(f_{\Lambda_{\mathrm{b}}}=58 \pm 10 \%\right)$ & \pm 0.04 \\
Background lepton fractions & \pm 0.03 \\
Effective background lifetime $\left(\tau_{\mathrm{b}}=1.537_{-0.021}^{+0.054} \mathrm{ps}\right)$ & +0.01 \\
$\Lambda_{\mathrm{b}} \rightarrow \ell$ physics function & \pm 0.03 \\
Background physics functions & \pm 0.03 \\
$\Lambda_{\mathrm{b}}$ polarisation $\left(\mathcal{P}_{\Lambda_{\mathrm{b}}}=-30_{-27}^{+32} \%\right)$ & \pm 0.03 \\
$\Lambda_{\mathrm{b}}$ decay model $(4-$ body decay $20 \pm 20 \%)$ & \pm 0.02 \\
Fragmentation $\left(\left\langle x_{\mathrm{b}}\right\rangle=0.714 \pm 0.012\right)$ & \pm 0.02 \\
Fragmentation $\Lambda$ spectrum $( \pm 10 \%)$ & \pm 0.02 \\
Decay background and Misid function & \pm 0.02 \\
Resolution function & \pm 0.02 \\
Level of combinatorial $\Lambda( \pm 15 \%)$ & \pm 0.01 \\
\hline Total & \pm 0.09 \\
\hline
\end{tabular}

Table 2: Contributions to the systematic uncertainty in the $\mathrm{b}$ baryon lifetime measurement using $\Lambda \ell^{-}$sample.

In Table 2, the various contributions to the systematic uncertainty in the b baryon lifetime measurement are listed in order of importance. The major contribution to the systematic error is from the uncertainty in the b baryon fraction $(58 \pm 10 \%)$. This uncertainty 
is dominated by the error in the estimate of the number of accidental combinations of $\Lambda \ell^{-}$ with respect to $\Lambda \ell^{+}$. The value for the ratio of accidental $\Lambda \ell^{-}$over the $\Lambda \ell^{+}$combinations used, $0.8 \pm 0.2$, contributes an error of ${ }_{-0.05}^{+0.04} \mathrm{ps}$ to the $\mathrm{b}$ baryon lifetime.

The relative fractions of the various background sources are obtained from a Monte Carlo simulation and their uncertainty leads to a systematic contribution of $\pm 0.03 \mathrm{ps}$ to the $\mathrm{b}$ baryon lifetime.

The physics functions depend on the average lifetimes of the $b$ and $c$ hadrons which populate the right-sign $\left(\Lambda \ell^{-}\right)$peak. The assumption that these have the same $\mathrm{b}$ hadron composition in $\mathrm{b} \rightarrow \ell$ sample as that used in measurements of the average $b$ hadron lifetime [14] is also considered as a source of systematic error. Simulation studies based on JETSET 7.3 have shown that the b baryon production is suppressed in events where a lepton is associated with a right-sign accidental $\Lambda$. This is the same effect which creates the asymmetry in the $\Lambda \ell^{-}-\Lambda \ell^{+}$accidental combinations. A variation in the effective background lifetime of ${ }_{-0.021}^{+0.054} \mathrm{ps}$, which covers the case of full suppression of $\mathrm{b}$ baryons, yields a systematic error of ${ }_{-0.03}^{+0.01} \mathrm{ps}$ on the $\mathrm{b}$ baryon lifetime. The error on the average $c$ lifetime has a negligible effect, due to the low charm background.

Another source of systematic uncertainty arises from the limited sample of simulated events used to parametrise the physics functions. The statistical uncertainties in the parameters of each physics function leads to an estimated error on b baryon lifetime of \pm 0.03 ps for the $\Lambda_{\mathrm{b}} \rightarrow \ell$ function and \pm 0.03 ps for the $\mathrm{b} \rightarrow \ell, \mathrm{b} \rightarrow \mathrm{c} / \tau \rightarrow \ell$ and $\mathrm{c} \rightarrow \ell$ combined.

An important systematic uncertainty arises from the lack of knowledge of the $\Lambda_{\mathrm{b}}$ polarisation. The $\Lambda_{\mathrm{b}} \rightarrow \ell$ physics function is sensitive to the polarisation of the $\Lambda_{\mathrm{b}}$, because the impact parameter of the lepton is correlated with its decay angle. In $\mathrm{Z} \rightarrow \mathrm{b} \overline{\mathrm{b}}$ decays, the b quark is produced with a longitudinal polarisation of $-94 \%$. b baryons can retain some fraction of this primordial polarisation. If the $\Lambda_{\mathrm{b}}$ is longitudinally polarised, the resulting lepton is produced preferentially along the $\Lambda_{\mathrm{b}}$ direction and oppositely to the polarisation vector. Therefore, due to the $\Lambda_{\mathrm{b}}$ boost, a left-hand polarisation of the $\Lambda_{\mathrm{b}}$ results, for the same lifetime, in a harder lepton spectrum and a smaller lepton impact parameter than if $\Lambda_{\mathrm{b}}$ were unpolarised. The uncertainty in the knowledge of the $\Lambda_{\mathrm{b}}$ polarisation $\left(\mathcal{P}_{\Lambda_{\mathrm{b}}}=-30_{-27}^{+32 \%}\right)$ [17], used in this analysis, leads to a systematic error of $\pm 0.03 \mathrm{ps}$.

A variation in the 4 -body semileptonic $b$ hadron decay rate (relative to the total $\mathrm{b}$ hadron semileptonic decay rate) of $\pm 20 \%$ leads to a change of $\pm 0.02 \mathrm{ps}$ in the $\mathrm{b}$ baryon lifetime. Uncertainties in the rate of different exclusive decay modes which make up the inclusive $\Lambda_{c}^{+} \rightarrow \Lambda \mathrm{X}$ rate gives a negligible contribution.

A further source of systematic error is due to the $b$ fragmentation. A variation of the Peterson fragmentation function which covers the uncertainty in the measurement of the $\mathrm{b}$ hadron momentum $\left(\left\langle x_{\mathrm{b}}\right\rangle=0.714 \pm 0.012\right)$ [19] leads to a \pm 0.02 ps variation in the $\mathrm{b}$ baryon lifetime.

The shape of the $\mathrm{b} \rightarrow \ell$ physics function is different for true $\Lambda$ 's produced during fragmentation and the combinatorial $\mathrm{p} \pi^{-}$combinations. In particular, a change in the $\Lambda$ momentum spectrum would affect the shape of the physics function. Therefore a further uncertainty arises in the simulation of the hadronization process which produces fragmentation $\Lambda$ 's. The momentum spectrum of $\Lambda$ 's produced in hadronic events simulated with JETSET 7.3 has been compared with the data [20] and shows a good agreement. However, an uncertainty of $10 \%$ in the shape of the fragmentation $\Lambda$ momentum spectrum is assumed, leading to an error of $0.02 \mathrm{ps}$ in the lifetime measurement.

The statistical error in the parametrisation of the misidentification and decay back- 
ground probability function is negligible. Nevertheless an error of $0.02 \mathrm{ps}$ is assigned to include possible uncertainties in the simulation of the decay background events used to derive the expected shape of the function.

The statistical error on the parametrisation of the resolution function gives a negligible uncertainty to the b baryon lifetime measurement. An error of $0.02 \mathrm{ps}$ is estimated from the variation in $b$ baryon lifetime when the uncorrected resolution function estimated using simulated events is replaced by the functional form corrected using data.

The number of combinatorial $\Lambda$ is obtained by interpolating the polynomial fit of the $\mathrm{p} \pi^{-}$invariant mass from the side bands to the region under the peak. A fit uncertainty of $15 \%$ results in a systematic contribution of $\pm 0.01 \mathrm{ps}$.

Summing all the systematic contributions in quadrature yields a total systematic error on the $\mathrm{b}$ baryon lifetime of $\pm 0.09 \mathrm{ps}$.

\section{Measurement of $\Lambda_{\mathrm{b}}^{0}$ lifetime using $\Lambda_{\mathrm{c}}^{+} \ell^{-}$correla- tion}

The $\Lambda_{\mathrm{c}}^{+} \ell^{-}$correlation is expected to have a large contribution from $\Lambda_{\mathrm{b}}^{0}$ semileptonic decay $\Lambda_{\mathrm{b}}^{0} \rightarrow \Lambda_{c}^{+} \ell^{-} \bar{\nu}$. The $\Lambda_{\mathrm{c}}^{+} \ell^{-}$combinations allow a measurement of the $\Lambda_{\mathrm{b}}^{0}$ decay vertex and hence its decay length on an event-by-event basis. It permits a complementary and independent measurement of the $\Lambda_{\mathrm{b}}^{0}$ lifetime.

\subsection{Selection of $\Lambda_{\mathrm{b}}^{0}$ using $\Lambda_{c}^{+} \ell^{-}$correlation}

Besides $\Lambda_{c}^{+} \ell^{-}$pairs from $\Lambda_{\mathrm{b}}^{0}$ semileptonic decay, two physics background processes can contribute to $\Lambda_{c}^{+} \ell^{-}$combinations in hadronic $\mathrm{Z}$ decays. These background sources are the same as the processes (2) and (3) discussed in section 3 . The third background component consists of combinatorial " $\Lambda_{c}^{+} \ell^{-}$" events, mainly a fake $\Lambda_{c}^{+}$associated with a real or a fake lepton.

Candidates for the decay $\Lambda_{\mathrm{b}}^{0} \rightarrow \Lambda_{c}^{+} \ell^{-} \bar{\nu}$ are identified in hadronic $\mathrm{Z}$ events where a $\Lambda_{c}^{+}$is associated with a lepton in the same hemisphere with respect to the thrust axis. The selection procedure is similar to that used in ref. [8]. Here the $\Lambda_{c}^{+}$candidates are reconstructed in three decay modes, namely, $\Lambda_{c}^{+} \rightarrow \mathrm{pK}^{-} \pi^{+}, \Lambda_{c}^{+} \rightarrow \Lambda \pi^{+} \pi^{+} \pi^{-}$and $\Lambda_{c}^{+} \rightarrow$ $\mathrm{p \overline {K } ^ { 0 }}$.

In the $\Lambda_{c}^{+} \rightarrow \mathrm{pK}^{-} \pi^{+}$channel, the proton, kaon and pion are required to have momenta greater than 4,2 and $1 \mathrm{GeV} / c$, respectively. The TPC $\mathrm{d} E / \mathrm{d} x$ measurement for proton candidates is required to be at least 2 standard deviations away from that expected for pions of similar momenta. For kaon (pion) candidates, the specific ionization is required, when available, to be within 2.5 (3) standard deviations of that expected. The $\Lambda_{c}^{+} \rightarrow$ $\Lambda \pi^{+} \pi^{+} \pi^{-}$candidates are selected by requiring the $\Lambda$ and the three pions to have momenta greater than 3 and $0.5 \mathrm{GeV} / c$, respectively. The $\Lambda$ candidates are selected by a procedure similar to that described in section 3 . In the $\Lambda_{c}^{+} \rightarrow \mathrm{pK}^{0}$ channel, the proton and the $\overline{\mathrm{K}}^{0}$ are required to have momenta greater than 3.5 and $2 \mathrm{GeV} / c$, respectively. The $\overline{\mathrm{K}}^{0}$ candidates are reconstructed in the channel $\mathrm{K}_{\mathrm{s}}^{0} \rightarrow \pi^{+} \pi^{-}$with the same algorithm as used for the $\Lambda$ selection. The invariant mass of the two pion candidates is required to be incompatible with the $\gamma$ and $\Lambda$ mass hypotheses. The cosine of the decay angle of the proton candidate in the $\mathrm{pK}_{\mathrm{s}}^{0}$ rest frame is required to be greater than -0.8 . The specific ionization for the two pions and the proton candidates, is required, when available, to be within 3 standard 
deviations of that expected for pions or protons. To remove reflections from $\mathrm{D}_{s}^{+} \rightarrow \mathrm{K}^{+} \mathrm{K}_{\mathrm{s}}^{0}$ and $\mathrm{D}^{+} \rightarrow \pi^{+} \mathrm{K}^{0} \mathrm{~s}$ decays, all combinations which are compatible with $\mathrm{D}_{\mathrm{s}}^{+}$or $\mathrm{D}^{+}$, using the appropriate mass hypothesis, are rejected.

The $\Lambda_{c}^{+}$candidates with momenta greater than $8 \mathrm{GeV} / c$ for $\Lambda_{c}^{+} \rightarrow \mathrm{pK}^{-} \pi^{+}$and $\Lambda_{c}^{+} \rightarrow \mathrm{pK}^{0}$ and $9 \mathrm{GeV} / c$ for $\Lambda_{c}^{+} \rightarrow \Lambda \pi^{+} \pi^{+} \pi^{-}$are combined with an identified lepton with momentum above $3 \mathrm{GeV} / c$ in a 45 degree cone around the $\Lambda_{c}^{+}$direction. The $\Lambda_{c}^{+} \ell^{-}$ combination is required to have a momentum greater than $20 \mathrm{GeV} / c$ and an invariant mass above $3.5 \mathrm{GeV} / c^{2}$. These two requirements reduce the combinatorial background and the contribution of physics background from non- $\Lambda_{\mathrm{b}}^{0}$ semileptonic decays.

A fit to the selected sample results in a sample of $57 \pm 11 \Lambda_{c}^{+} \ell^{-}$candidates (combinatorial background not included) of which $2.2 \pm 2.2$ combinations are attributed to physics background originating mainly from process (2). The $\Lambda_{c}^{+} \ell^{-}$reconstruction efficiency for each $\Lambda_{c}^{+}$decay channel is estimated from the JETseT 7.3 Monte Carlo. Using the measured branching ratios for the three $\Lambda_{c}^{+}$decay channels [14], this $\Lambda_{c}^{+} \ell^{-}$excess leads to an average product branching ratio

$$
\operatorname{Br}\left(\mathrm{b} \rightarrow \Lambda_{\mathrm{b}}^{0}\right) \cdot \operatorname{Br}\left(\Lambda_{\mathrm{b}}^{0} \rightarrow \Lambda_{\mathrm{c}}^{+} \ell^{-} \bar{\nu} \mathrm{X}\right)=(1.51 \pm 0.29 \text { (stat) } \pm 0.23(\text { syst })) \% .
$$

The measured rate is a sum over the electron and muon channels. The systematic error mainly reflects the uncertainty on the measured $\Lambda_{c}^{+}$branching ratios. It also contains the model dependence of the reconstruction efficiency and Monte Carlo statistics.

Table 3 summarises the number of $\Lambda_{c}^{+} \ell^{-}$signal combinations, the reconstruction efficiencies, and the product branching ratios obtained in the three $\Lambda_{c}^{+}$decay channels.

\begin{tabular}{|c|c|c|c|}
\hline Decay channel & Yield & Efficiency $(\%)$ & $B r\left(\mathrm{~b} \rightarrow \Lambda_{\mathrm{b}}\right) \cdot \operatorname{Br}\left(\Lambda_{\mathrm{b}} \rightarrow \Lambda_{c}^{+} \ell^{-} \bar{\nu} \mathrm{X}\right)$ \\
\hline \hline$\Lambda_{c}^{+} \rightarrow \mathrm{pK}^{-} \pi^{+}$ & $35.4 \pm 8.8$ & $7.9 \pm 0.5$ & $1.39 \pm 0.36 \pm 0.22 \%$ \\
$\Lambda_{c}^{+} \rightarrow \Lambda \pi^{+} \pi^{+} \pi^{-}$ & $10.2 \pm 4.5$ & $5.1 \pm 0.4$ & $1.65 \pm 0.76 \pm 0.37 \%$ \\
$\Lambda_{c}^{+} \rightarrow \mathrm{pK}^{0}$ & $11.7 \pm 4.0$ & $12.8 \pm 0.8$ & $1.81 \pm 0.64 \pm 0.38 \%$ \\
\hline \hline Total & $57.3 \pm 10.7$ & - & $1.51 \pm 0.29$ (stat) \pm 0.23 (syst)\% \\
\hline
\end{tabular}

Table 3: The number of $\Lambda_{\mathrm{c}}^{+} \ell^{-}$candidates and the $\operatorname{Br}\left(\mathrm{b} \rightarrow \Lambda_{\mathrm{b}}^{0}\right) \cdot \operatorname{Br}\left(\Lambda_{\mathrm{b}}^{0} \rightarrow \Lambda_{\mathrm{c}}^{+} \ell^{-} \bar{\nu} \mathrm{X}\right)$ values in the three $\Lambda_{c}^{+}$decay channels. The $\Lambda_{c}^{+} \ell^{-}$yields are the result of fits to the $\mathrm{pK}^{-} \pi^{+}, \Lambda \pi^{+} \pi^{+} \pi^{-}$and $\mathrm{pK}^{0}$ mass distributions using a Gaussian for the $\Lambda_{c}^{+}$peak and a first order polynomial for the combinatorial background.

\subsection{Measurement of $\Lambda_{\mathrm{b}}^{0}$ lifetime using $\Lambda_{\mathrm{c}}^{+} \ell^{-}$correlation}

The $\Lambda_{\mathrm{b}}^{0}$ lifetime measurement using $\Lambda_{c}^{+} \ell^{-}$correlation necessitates additional requirements on the track quality and vertexing for the decay length measurement. To ensure good reconstruction of the $\Lambda_{c}^{+}$and the $\Lambda_{b}^{0}\left(\Lambda_{c}^{+} \ell^{-}\right)$vertices, the lepton and at least two of the three tracks from the $\Lambda_{c}^{+} \rightarrow \mathrm{pK}^{-} \pi^{+}, \Lambda \pi^{+} \pi^{-} \pi^{+}$candidates are required to have one or more associated hits in the VDET and at least two hits in the ITC. For the $\Lambda_{c}^{+} \rightarrow \mathrm{pK}^{0}$ mode such requirement is made only on the lepton and the proton candidates. The $\chi^{2}$ probabilities of the $\Lambda_{c}^{+}$and the $\Lambda_{\mathrm{b}}^{0}$ vertices are both required to be greater than $1 \%$. Together with the $\Lambda_{c}^{+} \ell^{-}$selection cuts described in the previous section, these additional cuts yield 


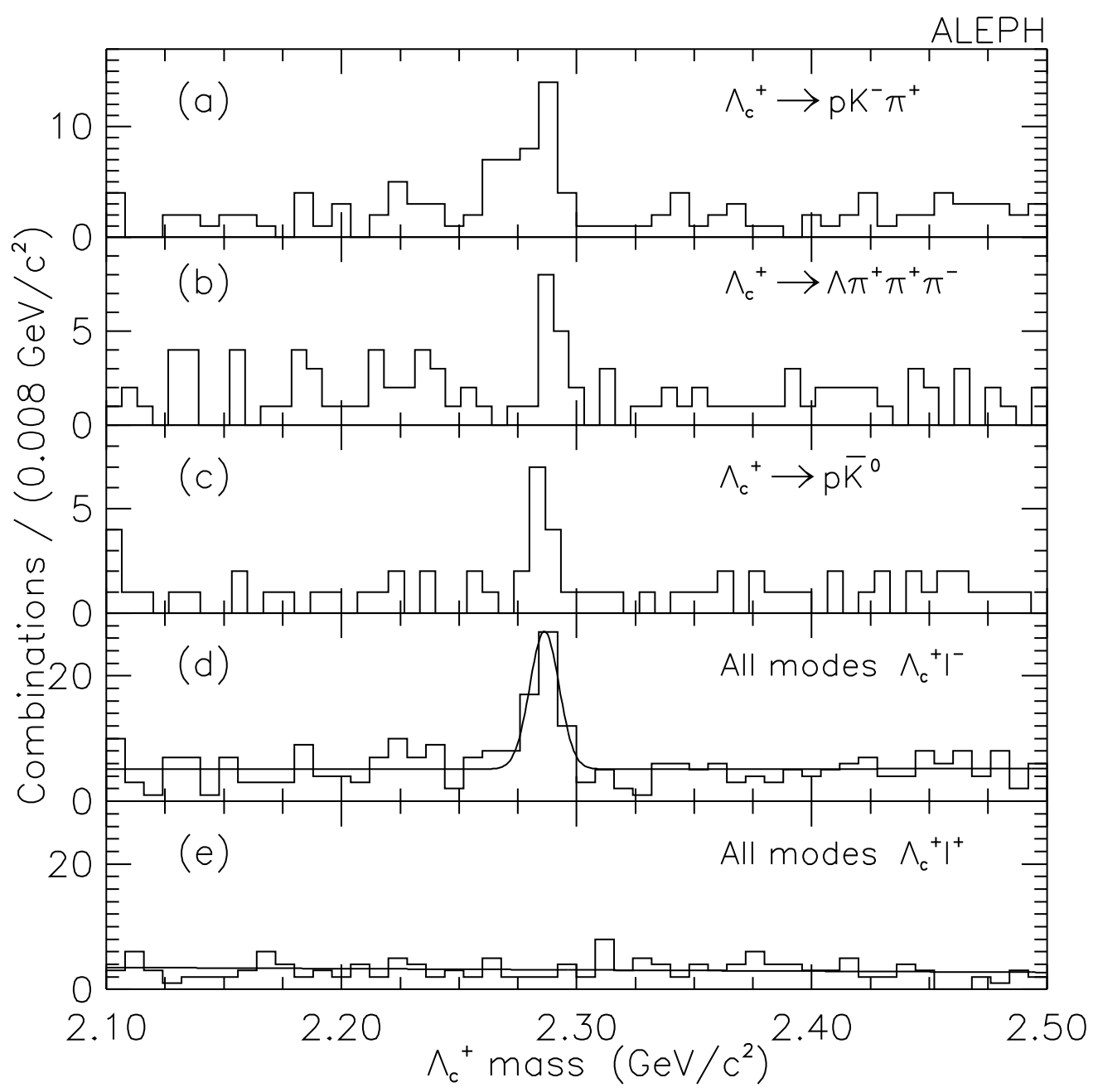

Figure 3: The $\mathrm{pK}^{-} \pi^{+}$(a), the $\Lambda \pi^{+} \pi^{+} \pi^{-}$(b), and the $\mathrm{pK}^{0}$ (c) invariant mass distributions for right-sign $\Lambda_{\mathrm{c}}^{+} \ell^{-}$combinations and their sum (d). The sum of wrong-sign $\Lambda_{\mathrm{c}}^{+} \ell^{+}$combinations is displayed in (e).

a total of $58 \Lambda_{c}^{+} \ell^{-}$combinations selected in a $\pm 2 \sigma$ window around the nominal $\Lambda_{c}^{+}$mass. Fig. 3 shows the individual contributions of the three $\Lambda_{c}^{+}$decay channels to the right-sign $\Lambda_{c}^{+} \ell^{-}$combinations and their sum after all cuts. A clear enhancement is observed at the nominal $\Lambda_{c}^{+}$mass value in the right-sign $\Lambda_{c}^{+} \ell^{-}$combinations while for wrong-sign $\Lambda_{c}^{+} \ell^{+}$ events (Fig. 3e) no such enhancement is observed. Table 4 summarises the number of $\Lambda_{c}^{+} \ell^{-}$candidates selected in the three $\Lambda_{c}^{+}$decay channels. The fraction of combinatorial background within the same mass window results from a first order polynomial fit to the invariant mass distributions. From Monte Carlo simulations, the expected number of $\Lambda_{c}^{+} \ell^{-}$combinations from process (2) is $1.5 \pm 1.5$. The corresponding effect on the lifetime measurement is quoted as a systematic uncertainty. 


\begin{tabular}{|c|c|c|c|}
\hline Decay Channel & $N_{\text {peak }}$ & $f_{\text {back }}(\%)$ & Mass Resolution $\left(\mathrm{MeV} / \mathrm{c}^{2}\right)$ \\
\hline \hline$\Lambda_{c}^{+} \rightarrow \mathrm{pK}^{-} \pi^{+}$ & 31 & $23 \pm 3$ & $6.9 \pm 0.4$ \\
$\Lambda_{c}^{+} \rightarrow \Lambda \pi^{+} \pi^{+} \pi^{-}$ & 13 & $24 \pm 5$ & $5.3 \pm 0.4$ \\
$\Lambda_{c}^{+} \rightarrow \mathrm{pK}^{0}$ & 14 & $25 \pm 4$ & $8.4 \pm 0.3$ \\
\hline \hline Total & 58 & - & - \\
\hline
\end{tabular}

Table 4: The number of $\Lambda_{c}^{+} \ell^{-}$candidates $N_{\text {peak }}$ and the fraction $f_{\text {back }}$ of combinatorial background within $\pm 2 \sigma$ of the nominal $\Lambda_{c}^{+}$mass in the three $\Lambda_{c}^{+}$decay modes after all requirements. The invariant mass resolution for each decay mode is also indicated.

\subsubsection{Measurement of $\Lambda_{\mathrm{b}}^{0}$ proper decay time}

The $\Lambda_{\mathrm{b}}^{0}$ lifetime is determined from the proper decay time distributions of the three $\Lambda_{\mathrm{c}}^{+} \ell^{-}$ event samples. For each $\Lambda_{\mathrm{b}}^{0}$ candidate, the proper time is obtained from the $\Lambda_{\mathrm{b}}^{0}$ decay length $l$, the $\Lambda_{\mathrm{b}}^{0}$ momentum $p$, and the $\Lambda_{\mathrm{b}}^{0}$ mass $\mathrm{M}$

$$
t_{\Lambda_{\mathrm{b}}^{0}}=\frac{l \mathrm{M}}{p}
$$

The $\Lambda_{\mathrm{b}}^{0}$ decay length is measured in three dimensions by projecting the vector joining the interaction point and the $\Lambda_{\mathrm{b}}^{0}$ decay vertex onto the $\Lambda_{\mathrm{b}}^{0}$ direction of flight as estimated from the $\Lambda_{c}^{+} \ell^{-}$combinations. From the Monte Carlo simulation, the resolution of the $\Lambda_{c}^{+}$and the $\Lambda_{\mathrm{b}}^{0}$ decay vertices along their directions of flight are $330 \mu \mathrm{m}$ and $180 \mu \mathrm{m}$ respectively. For each event, the error on the $\Lambda_{\mathrm{b}}^{0}$ decay length is calculated from the track trajectory errors. To estimate the accuracy of these errors, a distribution of the measured decay length divided by its uncertainty is built for fake " $\Lambda_{c}^{+} \ell^{-}$" events (with zero decay length) selected in a data sample of light quark enriched events. The negative side of this distribution is fitted with a Gaussian whose sigma is found to be $10 \%$ larger than unity, showing that the measured decay length errors are underestimated. Therefore, the measured $\Lambda_{\mathrm{b}}^{0}$ decay length error is increased by $10 \%$.

The $\Lambda_{\mathrm{b}}^{0}$ momentum is defined as $p=\sqrt{\left(E_{\Lambda_{c}^{+} \ell^{-}}+E_{\nu}\right)^{2}-\mathrm{M}^{2}}$ where $E_{\Lambda_{c}^{+} \ell^{-}}$is the measured $\Lambda_{c}^{+} \ell^{-}$energy and $E_{\nu}$ is the reconstructed neutrino energy in the $\Lambda_{c}^{+} \ell^{-}$hemisphere. The technique used to reconstruct the missing neutrino energy is similar to that described in ref. [21]. The neutrino energy measured by this method is overestimated on average by $2.4 \mathrm{GeV}$ due to uncounted neutral hadron energy. To take into account the uncertainty on the $\Lambda_{\mathrm{b}}^{0}$ momentum reconstruction, a correction distribution $\kappa$ determined from Monte Carlo simulation and defined as the ratio of the reconstructed to the true $\Lambda_{\mathrm{b}}^{0}$ momenta is introduced for each $\Lambda_{c}^{+} \ell^{-}$sample. The $\kappa$ distribution, is on average, centered at 1.07 with an r.m.s. of $10.4 \%$.

For the $\Lambda_{\mathrm{b}}^{0}$ mass, the quark model prediction of $\mathrm{M}=5.6 \mathrm{GeV} / c^{2}[6]$ is used.

\subsubsection{The fitting procedure and result of the fit}

The $\Lambda_{\mathrm{b}}^{0}$ lifetime is extracted from a simultaneous unbinned maximum likelihood fit to the proper time distribution of the three $\Lambda_{c}^{+} \ell^{-}$event samples. The fitting technique is similar to that described in [21]. The event samples used to extract the $\Lambda_{\mathrm{b}}^{0}$ lifetime are shown in Table 4. The total sample consists of $N_{\text {peak }} \Lambda_{c}^{+} \ell^{-}$events, in which a fraction 
$f_{\text {back }}$ is attributed to combinatorial background events. To determine the background parametrisation for each $\Lambda_{c}^{+} \ell^{-}$event sample, right-sign events from side bands more than $4 \sigma$ outside the nominal $\Lambda_{c}^{+}$mass and wrong-sign events $\left(N_{\text {back }}\right)$ are included in the fit. The likelihood function $\mathcal{L}$ used is the following

$$
\mathcal{L}=\prod_{k=1}^{3}\left(\prod_{i=1}^{N_{\text {peak }}^{(k)}}\left\{\left(1-f_{\text {back }}^{(k)}\right) \mathcal{P}_{\text {sig }}\left(t_{i, k}, \tau_{\Lambda_{\mathrm{b}}^{0}}\right)+f_{\text {back }}^{(k)} \mathcal{P}_{\text {back }}\left(t_{i, k}\right)\right\} \times \prod_{j=1}^{N_{\text {back }}^{(k)}} \mathcal{P}_{\text {back }}\left(t_{j, k}\right)\right)
$$

The probability density function $\mathcal{P}_{\text {sig }}$ of the $\Lambda_{\mathrm{b}}^{0}$ signal consists of an exponential function of lifetime $\tau_{\Lambda_{\mathrm{b}}^{0}}$, convoluted with the resolution function discussed above and with the $\kappa$ distribution. The probability density function $\mathcal{P}_{\text {back }}$ for combinatorial background events consists of two components: one has zero lifetime and the other is an exponential function with non-zero lifetime. Each component is convoluted with the resolution function. Fig. 4 shows the result of the simultaneous fit of the $\Lambda_{\mathrm{b}}^{0}$ signal and combinatorial background events. The fitted $\Lambda_{\mathrm{b}}^{0}$ lifetime is

$$
\tau_{\Lambda_{\mathrm{b}}^{0}}=1.02+0.23 \mathrm{ps}
$$

where the quoted error is statistical.

\subsection{Systematic errors}

Various sources of systematic uncertainties in $\tau_{\Lambda_{b}}$ measurement have been considered. Their contributions are summarised in Table 5 .

\begin{tabular}{|l|c|}
\hline Source & Uncertainty (ps) \\
\hline \hline Combinatorial background fraction & \pm 0.01 \\
Combinatorial background shape & \pm 0.04 \\
Physics background $(1.5 \pm 1.5$ events $)$ & \pm 0.03 \\
Resolution function & \pm 0.01 \\
$\Lambda_{\mathrm{b}}^{0}$ decay model $(20 \% \pm 20 \%$ four-body) & \pm 0.02 \\
$\mathrm{~b}$ fragmentation & \pm 0.01 \\
$\Lambda_{\mathrm{b}}^{0}$ polarization $\left(\mathcal{P}_{\Lambda_{\mathrm{b}}^{0}}=-30_{-27}^{+32} \%\right)$ & $\leq 0.01$ \\
$E_{\nu}$ calibration & $\leq 0.01$ \\
$\Lambda_{\mathrm{b}}^{0}$ mass $(5.6 \pm 0.1) \mathrm{GeV} / c^{2}$ & \pm 0.02 \\
\hline \hline total & 0.06 \\
\hline
\end{tabular}

Table 5: Sources of systematic uncertainty in the $\Lambda_{\mathrm{b}}^{0}$ lifetime

Varying the number of combinatorial background events in the peak within its statistical error leads to a $\Lambda_{\mathrm{b}}^{0}$ lifetime change of \pm 0.01 ps. Using an alternative parametrisation of the proper time distribution of background events leads to a $\Lambda_{\mathrm{b}}^{0}$ lifetime change of 0.04 ps.

The contribution of background process $\mathrm{B} \rightarrow \Lambda_{c}^{+} X \ell^{-} \nu$ to the number of right-sign events in the peak is $1.5 \pm 1.5$. Adding an exponential component to the background probability density function to reproduce the possible lifetime bias introduced by these events leads to a $\Lambda_{\mathrm{b}}^{0}$ lifetime variation of $\pm 0.03 \mathrm{ps}$. 


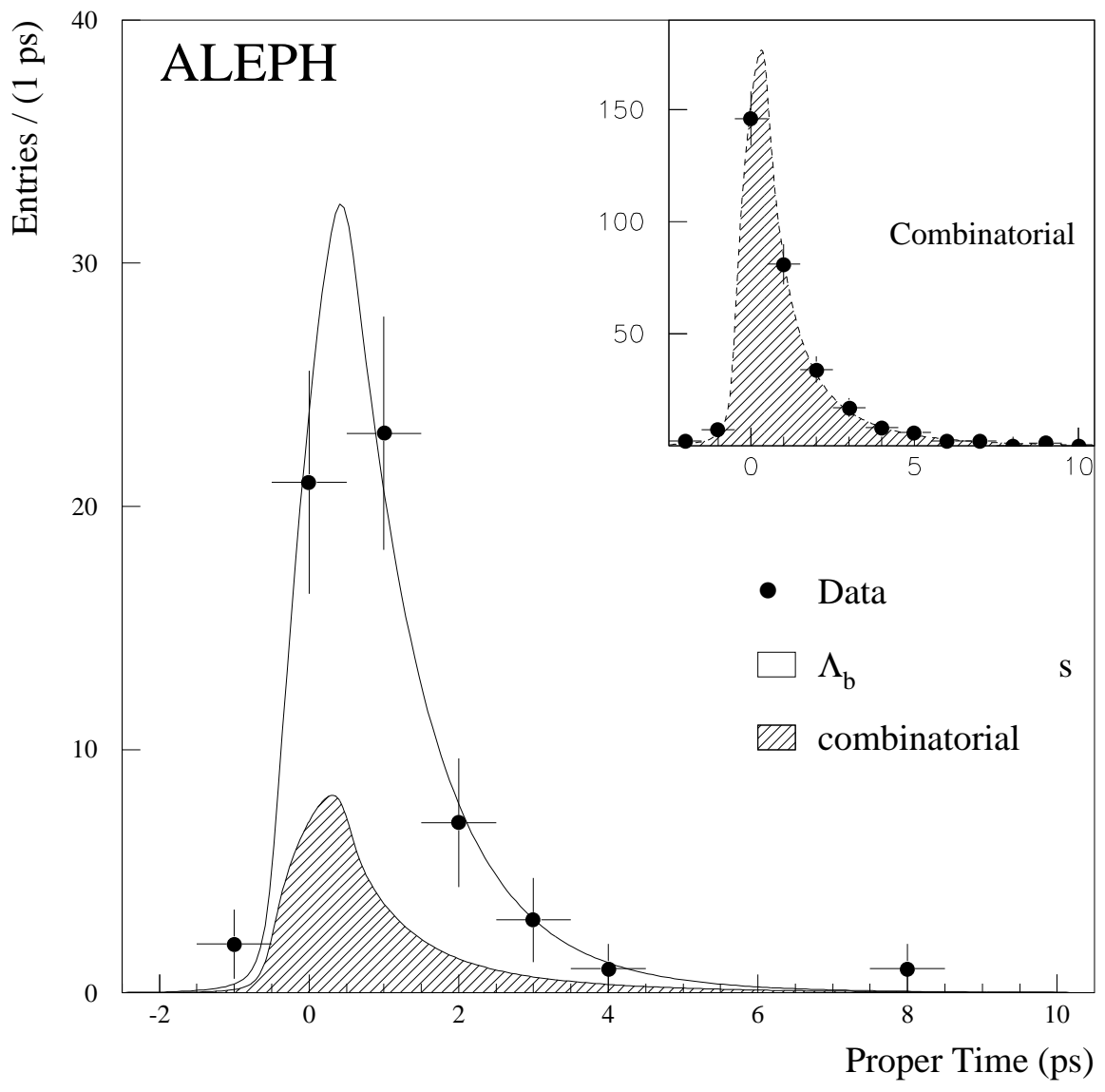

Figure 4: The proper-time distribution of the $\Lambda_{\mathrm{b}}^{0}$ candidates in the $\Lambda_{\mathrm{c}}^{+} \ell^{-}$sample. The shaded area corresponds to the proper-time distribution of the combinatorial background. The solid line is the result of the maximum likelihood fit described in the text. The inset shows the proper time distribution of the combinatorial background from wrong-sign events and events in right-sign sidebands. The fraction of combinatorial background events with zero lifetime differs among the $3 \Lambda_{c}^{+}$decay modes. It is $27 \%$ on average.

To estimate the systematic error due to the decay length resolution function, an alternative parametrisation consisting of two Gaussians is used. Varying the widths of the Gaussian by $\pm 20 \%$ and the relative fraction of the second Gaussian from 0 to 0.2 changes the $\Lambda_{\mathrm{b}}^{0}$ lifetime by \pm 0.01 ps.

The final state multiplicity in $\Lambda_{\mathrm{b}}^{0}$ semileptonic decay may consist of four or more particles, though by analogy with $\mathrm{B}$ mesons three-body decays should dominate. Using $(20 \pm 20) \%$ four-body decays such as $\Lambda_{\mathrm{b}}^{0} \rightarrow \Lambda_{c}^{+} \ell^{-} \bar{\nu} \pi^{0} / \rho^{\circ}$ yields a $\Lambda_{\mathrm{b}}^{0}$ lifetime change of \pm 0.02 ps.

The $\kappa$ distribution depends on Monte Carlo inputs such as the fragmentation scheme [22] and the $\Lambda_{\mathrm{b}}^{0}$ polarization [17]. The effects of these inputs on the $\Lambda_{\mathrm{b}}^{0}$ lifetime are small. Particularly, the $\Lambda_{\mathrm{b}}^{0}$ polarization has no effect due to the use of the reconstructed neutrino 
energy. To estimate the systematic error on $\Lambda_{\mathrm{b}}^{0}$ lifetime due to the neutrino energy reconstruction error, the relative proportion of events in the tail of the $\kappa$ distribution is varied by $20 \%$. This introduces a negligible change in the $\Lambda_{\mathrm{b}}^{0}$ lifetime.

The uncertainty on the $\Lambda_{\mathrm{b}}^{0}$ mass $(5.6 \pm 0.1) \mathrm{GeV} / c^{2}$ taken from theoretical predictions [6] leads to a $\Lambda_{\mathrm{b}}^{0}$ lifetime uncertainty of $\pm 0.02 \mathrm{ps}$.

Combining the systematic errors from the aforementioned sources, the total systematic error is \pm 0.06 ps.

\section{Conclusion}

From a total of about 1.5 million hadronic $\mathrm{Z}$ decays collected with the ALEPH detector between 1991 and 1993, the yields of $\Lambda \ell^{-}$and $\Lambda \ell^{+}$combinations are measured. Semileptonic decays of b baryon result in a signal of $290 \pm 35$ (stat) \pm 39 (syst) $\Lambda \ell^{-}$combinations, which corresponds to a product branching ratio of

$$
\operatorname{Br}\left(\mathrm{b} \rightarrow \Lambda_{\mathrm{b}}\right) \cdot \operatorname{Br}\left(\Lambda_{\mathrm{b}} \rightarrow \Lambda_{\mathrm{c}}^{+} \ell^{-} \bar{\nu} \mathrm{X}\right) \cdot \operatorname{Br}\left(\Lambda_{\mathrm{c}}^{+} \rightarrow \Lambda \mathrm{X}\right)=(0.61 \pm 0.07 \text { (stat) } \pm 0.10 \text { (syst) }) \% .
$$

The lifetime of $b$ baryons is measured from a maximum likelihood fit to the impact parameter distribution of the candidate lepton tracks in the $\Lambda \ell^{-}$sample. The result is

$$
\tau_{\text {b-baryon }}=1.05_{-0.11}^{+0.12}(\text { stat }) \pm 0.09(\text { syst }) \text { ps. }
$$

$\Lambda_{c}^{+} \ell^{-}$combinations from semileptonic decay of the $\Lambda_{\mathrm{b}}^{0}$ are also identified via fully reconstructed $\Lambda_{c}^{+} \rightarrow \mathrm{pK}^{-} \pi^{+}, \Lambda_{c}^{+} \rightarrow \Lambda \pi^{+} \pi^{+} \pi^{-}$, and $\Lambda_{c}^{+} \rightarrow \mathrm{pK}^{0}$ decays. The average product branching ratio is

$$
\operatorname{Br}\left(\mathrm{b} \rightarrow \Lambda_{\mathrm{b}}^{0}\right) \cdot \operatorname{Br}\left(\Lambda_{\mathrm{b}}^{0} \rightarrow \Lambda_{\mathrm{c}}^{+} \ell^{-} \bar{\nu} \mathrm{X}\right)=(1.51 \pm 0.29 \text { (stat) } \pm 0.23 \text { (syst) }) \% .
$$

The $\Lambda_{\mathrm{b}}^{0}$ lifetime measured from a maximum likelihood fit of the proper decay time distribution of $58 \Lambda_{c}^{+} \ell^{-}$combinations is

$$
\tau_{\Lambda_{\mathrm{b}}^{0}}=1.02_{-0.18}^{+0.23} \text { (stat) } \pm 0.06(\text { syst }) \text { ps. }
$$

Since the relative contribution of various b baryon species in the $\Lambda \ell^{-}$sample is not well known and depends on their relative production rate and lifetime, there is no a priori prescription for averaging the two lifetime measurements described above. Assuming that the $\Lambda_{\mathrm{b}}^{0}$ is the dominant source of b baryons produced at the $\mathrm{Z}$ resonance and the differences among the lifetimes of $\mathrm{b}$ baryons $\left(\Lambda_{\mathrm{b}}^{0}, \Xi_{\mathrm{b}}, \Omega_{\mathrm{b}}\right)$ are small, the two measurements can be averaged ${ }^{4}$ to give:

$$
\tau_{\Lambda_{\mathrm{b}}^{0}}=1.04_{-0.12}^{+0.13} \mathrm{ps}
$$

This value can be compared with the world average of the $\mathrm{B}^{0}$ lifetime [23] of $1.61 \pm$ $0.09 \mathrm{ps}$ and is displayed in Fig. 5. The ratio of the lifetimes of $\Lambda_{\mathrm{b}}^{0}$ and $\mathrm{B}^{0}$ hadrons is $0.65 \pm 0.09$ which is substantially smaller than the theoretical estimate [1] of 0.9 . This difference between the experimental measurement and the theoretical prediction remains to be understood.

\footnotetext{
${ }^{4} \mathrm{~A}$ small correlation of $1.3 \%$ between the $\Lambda \ell^{-}$and $\Lambda_{c}^{+} \ell^{-}$samples has been ignored in the averaging process.
} 


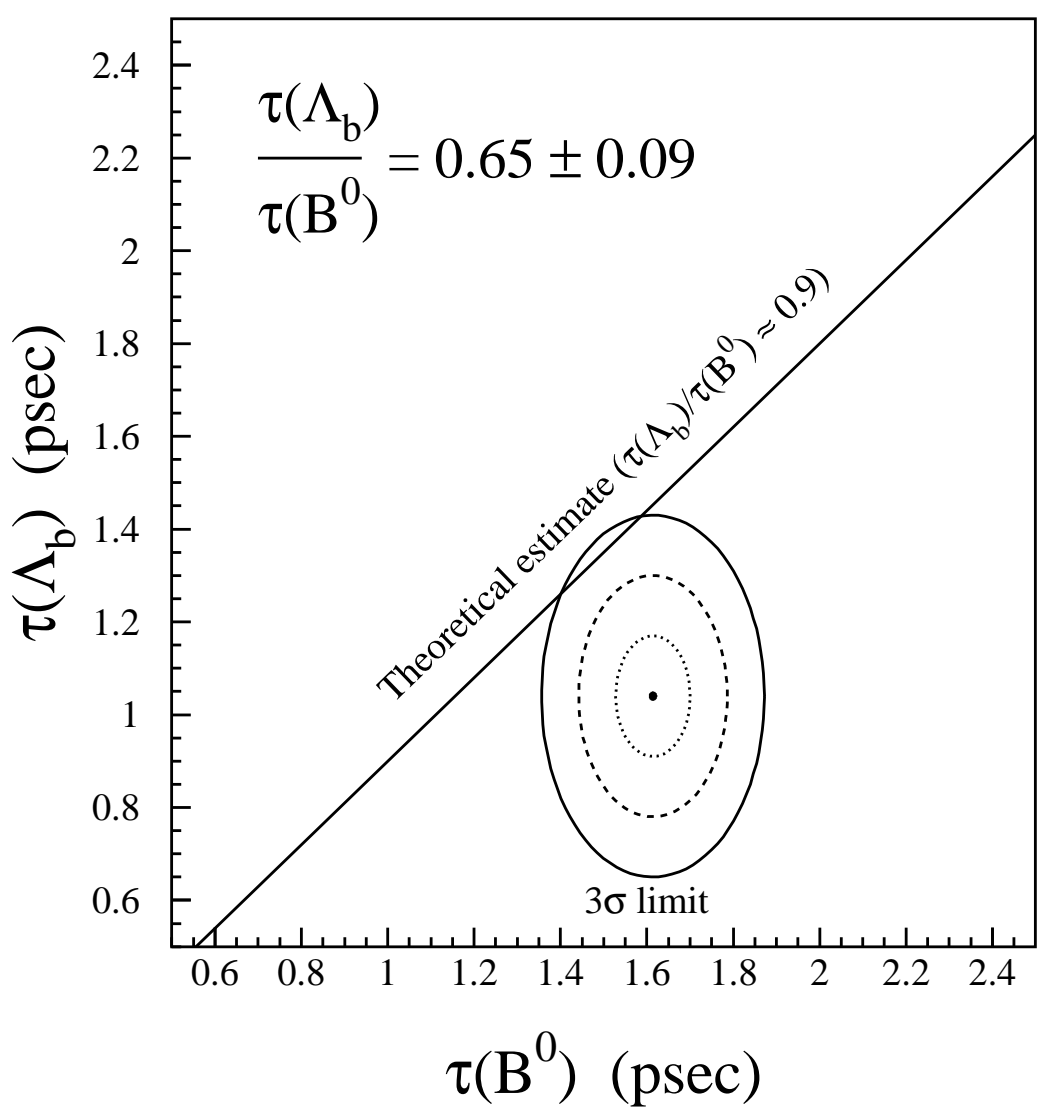

Figure 5: Comparison of the $\Lambda_{\mathrm{b}}$ lifetime $\left(\tau_{\Lambda_{\mathrm{b}}}=1.04_{-0.12}^{+0.13} \mathrm{ps}\right)$ reported in this letter with the world average of the $\mathrm{B}^{0}$ lifetime $\left(\tau_{\mathrm{B}^{0}}=1.61 \pm 0.09 \mathrm{ps}\right)$. The one, two and three standard deviation contours are shown as dotted, dashed and solid line respectively. The straight line represents the theoretical estimate [1] of the ratio of the $\Lambda_{\mathrm{b}}^{0}$ and $\mathrm{B}^{0}$ lifetimes.

\section{Acknowledgements}

We would like to thank T. Sjöstrand and G. Schuler for useful discussions and assistance regarding the modelling of $b$ polarization in $\mathrm{Z}$ decays. We thank our colleagues in the CERN accelerator division for the successful operation of the LEP storage ring. We also thank the engineers and technicians in all our institutions for their support in constructing and operating ALEPH. Those of us from non-member countries thank CERN for its hospitality.

\section{References}

[1] I. Bigi et al., Non-leptonic Decays of Beauty Hadrons - From Phenomenology to Theory (in "B decays", second edition, published by World Scientific, Singapore). 
[2] D. Buskulic et al., (Aleph Collab.), Phys. Lett. B307 (1993) 194; Phys. Lett. B322 (1994) 275;

P. Abreu et al., (Delphi Collab.), Z. Phys. C57 (1993) 181; Z. Phys. C61 (1994) 407 ;

P.D. Acton et al., (opal Collab.), Phys. Lett. B307 (1993) 247; Phys. Lett. B312 (1993) 501.

[3] F. Abe et al., cDF Collab., Phys. Rev. Lett. 72 (1994) 3456.

[4] D. Buskulic et al., (A Leph Collab.), Phys. Lett. B297 (1992) 449;

P. Abreu et al., (Delphi Collab.), Phys. Lett. B311 (1993) 379;

P.D. Acton et al. (opal Collab.), Phys. Lett. B316 (1993) 435.

[5] D. Buskulic et al., (A Leph Collab.), Phys. Lett. B278 (1992) 209;

P. Abreu et al., (Delphi Collab.), Phys. Lett. B311 (1993) 379;

P.D. Acton et al., (opal Collab.), Phys. Lett. B281 (1992) 394.

[6] A. Martin and J.-M. Richard, Phys. Lett. B185 (1987) 426.

[7] W. Kwong and J. Rosner, Phys. Rev. D44 (1991) 212.

[8] D. Buskulic et al., (A Leph Collab.), Phys. Lett. B294 (1992) 145.

[9] D. Decamp et al., (Aleph Collab.), Nucl. Inst. and Meth. A294 (1990) 121;

[10] G. Batignani et al., Conference Record of the 1991 IEEE Nucl. Science Symp., 1991, Santa Fe, New Mexico, USA.

[11] D. Buskulic et al., (ALEPH Collab.), CERN-PPE/94-170.

[12] D. Buskulic et al., (a leph Collab.), Nucl. Inst. and Meth. A346 (1994) 461.

[13] H. Albrecht et al., (A Rgus Collab.), Phys. Lett. B249 (1990) 359.

[14] Particle Data Group, Phys. Rev. D50 (1994) 1173.

[15] T. Sjöstrand and M. Bengtsson, Comp. Phys. Com. 46 (1987) 43.

[16] T. Mannel and G.A. Schuler, Phys. Lett. B279 (1992) 194.

[17] D. Buskulic et al., (ALePh Collab.), Measurement of the $\Lambda_{\mathrm{b}}$ polarization at LEP, Contribution to the 27th International Conference on High Energy Physics, Glasgow, Scotland, 20-27 July 1994.

[18] D. Buskulic et al., (A teph Collab.), Phys. Lett. B313 (1993) 535.

[19] D. Buskulic et al., (Aleph Collab.), Z. Phys. C62 (1994) 179.

[20] D. Buskulic et al., (A Leph Collab.), Z. Phys. C64 (1994) 361.

[21] D. Buskulic et al., (A ateph Collab.), Phys. Lett. B 322 (1994) 275.

[22] C. Peterson et al., Phys. Rev. D27 (1983) 105.

[23] P. Roudeau, Plenary talk on "Heavy Quark Physics" at the 27th International Conference on High Energy Physics, Glasgow, Scotland, 20-27 July 1994. 